\title{
A Systematic Error in X-ray Grating Interferometry due to Asymmetric Scattering Distributions
}

\author{
Peter Modregger ${ }^{*, \dagger}$, Bernd Rudolf Pinzer* and Marco Stampanoni ${ }^{*, * *}$ \\ * Swiss Light Source, Paul Scherrer Institut, Villigen, Switzerland \\ $\dagger$ School of Biology and Medicine, University of Lausanne, Lausanne, Switzerland \\ ** Institute for Biomedical Engineering, UZH/ETH Zürich, Zürich, Switzerland
}

\begin{abstract}
The high sensitivity provided by x-ray grating interferometry is one of the distinct characteristics of this phase contrast imaging technique. Up to now, several contributions to the uncertainty of the determined phase signal are known, which can be addressed by adapting total acquisition time or improving the mechanics of the experimental setup. Here, we demonstrate the occurrence of an additional systematic error, which is intrinsic to the physics of the contrast formation process. Based on a recently established scattering-based description of grating interferometry, we demonstrate analytically that asymmetric scattering distributions lead to a systematic error in the determined phase signal.
\end{abstract}

Keywords: grating interferometry, $\mathrm{x}$-ray imaging, data analysis, systematic errors

PACS: 87.59.-e,07.60.Ly,07.85.Qe,42.30.Rx

\section{INTRODUCTION}

Since its first experimental demonstration [1, 2] grating interferometry (GI) - a phasesensitive, hard x-ray imaging technique - has attracted increasing interest from the field of biomedical research [3-5]. An important contribution to this interest is the particular high sensitivity towards electron-density variations in the sample [6], which renders GI especially suitable for imaging subtle differences in soft tissue materials. The high sensitivity is realized by taking advantage of phase contrast, which is, in general, orders of magnitude stronger than absorption contrast in the hard X-ray regime [7].

GI utilizes a beam splitter and an analyzer line grating in order to facilitate phase sensitivity (Fig. 1). The beam splitter introduces a periodical phase shift into the incident wave, which provides a rectangular-shaped interference pattern at certain distances [8]. Refraction taking place at the sample will lead to a lateral offset of this interference pattern. Since refraction is proportional to the first derivative of the phase signal after transmission through the sample [9], sensitivity to phase is achieved. However, the typical period of the rectangular-shaped interference pattern is in the order of a few microns, which is too small to be resolved by standard x-ray imaging detectors. Thus, an additional absorption grating is introduce in front of the detector in order to analyze the lateral offset of the interference pattern. By scanning either of the gratings, an oscillatory intensity pattern is recorded in each pixel, which is called the phase stepping curve (PSC). The PSC can be analyzed by a Fourier-based approach in order to retrieve the three complementary contrast, which are provided by GI: absorption, phase and dark- 


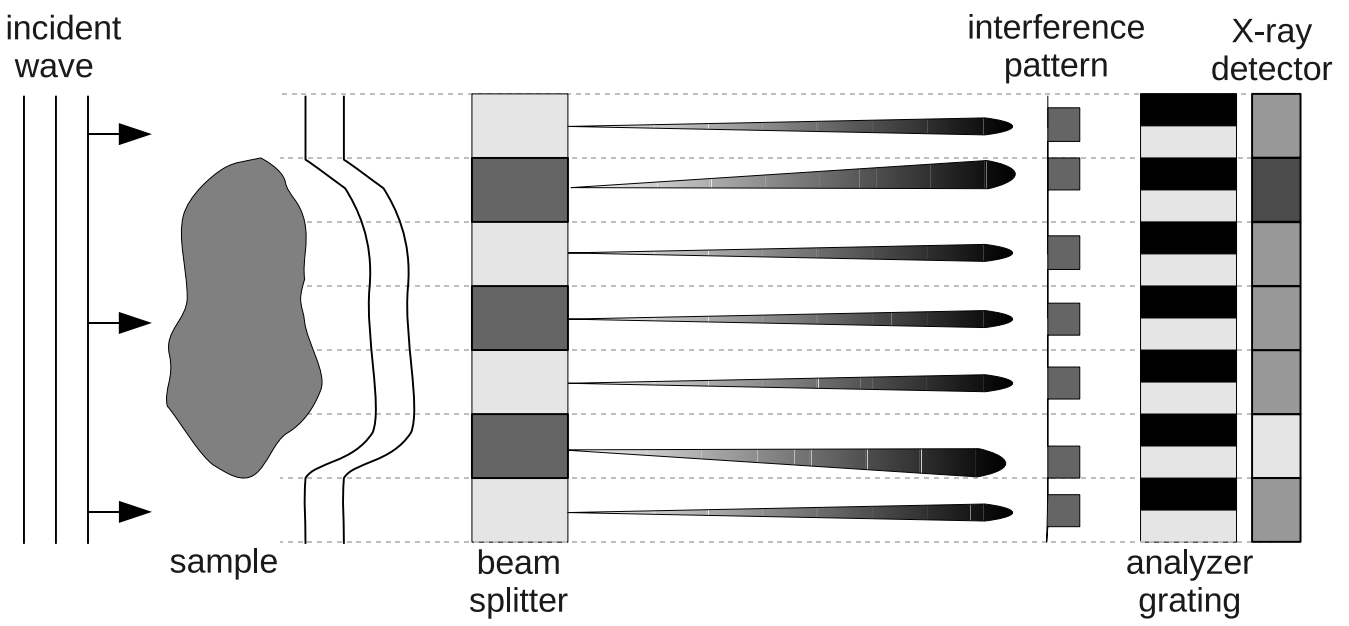

FIGURE 1. Sketch of the grating interferometer.

field contrast [9-11]. More details about the contrast formation process, data analysis procedures and experimental implementation can be found in literature $[9,12,13]$.

\section{UNCERTAINTY OF THE PHASE SIGNAL}

It is clear that a thorough understanding of all possible contributions to the uncertainty of the determined phase signal is crucial for maximizing the performance of GI. The known contributions can be best understood by looking on the details of the experimental scan and data analysis procedures.

In experiment, a flat-field PSC, $f\left(\phi_{m}\right)$, without the sample and a sample PSC, $s\left(\phi_{m}\right)$, is obtained, where $\phi_{m}$ denotes the lateral offset of step $m$ during the scan in radians. Since the PSCs are periodical, their intensity distributions may be expressed by a complex Fourier series [12]

$$
s\left(\phi_{m}\right)=\sum_{n} a_{n} \exp \left(-i\left(q_{n} \phi_{m}+P\right)\right) .
$$

$a_{n}$ are the Fourier coefficients, $q_{n}$ are the spatial frequencies and $P$ constitutes the to be determined fringe offset. The flat-field PSC is described by eq. (1) for $P=0 . P$ can be determined by calculating the difference of the phase of the first non-trivial Fourier component $q_{1}$ of the Fourier transforms of the individual PSCs

$$
P=\arg \left(\hat{s}\left(q_{1}\right)\right)-\arg \left(\hat{f}\left(q_{1}\right)\right) .
$$

The caret denotes the Fourier transform of the corresponding functions.

At least three contributions to the uncertainty of $P$ have been published.

- Phase step jitter, which reflects mechanical instabilities of the experimental setup and can be regarded as an uncertainty in the position of the phase step $\phi_{m}$ in eq. (1). If Piezo actuators are utilized for scanning the grating, this contribution is usually negligible $[14,15]$. 
- Photon statistics, which takes into account the statistical nature of intensity measurements and can be incorporated into eq. (1) by an uncertainty of the intensities $s\left(\phi_{m}\right)$ or $f\left(\phi_{m}\right)$. This contribution has been studied in depth and has been identified as the main influence on the uncertainty of the phase signal [14-17].

- "Anatomical noise", which relates to the fact that for a finite number of phase steps $P$ is only retrieved exactly by eq. (2) if $a_{n}=0$ for $n>1$ [17]. In other words, the uncertainty of $P$ depends on the exact shape of the PSCs. This effect decreases with an increasing number of phase steps.

If delivered dose is not an issue, all these contributions can be addressed by either increasing the total number of acquired photons or improving the mechanical stability of the experimental setup.

\section{INFLUENCE OF ASYMMETRIC SCATTERING DISTRIBUTIONS}

Recently, we established an alternative perspective on the contrast formation process of GI, which is based on scattering [18]. In the following, we will show that this alternative view offers the possibility to identify an additional error in the retrieval of the phase signal.

The scattering distribution $g(\phi)$ can be regarded as the histogram of all refraction angles contributing to the signal in one detector pixel. $g(\phi)$ relates to the PSCs by [11]

$$
s(\phi)=f(\phi) \otimes g(\phi) .
$$

Inserting eq. (3) into eq. (2)

$$
P=\arg \left(\hat{f}\left(q_{1}\right) \cdot \hat{g}\left(q_{1}\right)\right)-\arg \left(\hat{f}\left(q_{1}\right)\right)=\arg \left(\hat{g}\left(q_{1}\right)\right)
$$

reveals that the value retrieved by the standard analysis procedure, $P$, is equal to the phase of the Fourier transform of the scattering distribution at the appropriate spatial frequency.

We associated the complementary contrasts (i.e., absorption, phase and dark-field), which are delivered by grating interferometry, with the orders $l$ of the (centralized) moments $M_{l}$ of the scattering distribution [18]. The centralized moments are defined by [19]

$$
M_{l}=\int d \phi(\phi-\mu)^{n} g(\phi) .
$$

$\mu$ is the expectation value of the distribution $g(\phi)$ and represents the to-be-determined fringe offset in a least-squares sense. Ideally, the data analysis procedure (eq. 2) should deliver $P=\mu$. However, this will turn out to be true only under certain conditions.

In Fourier space the centralized moments $M_{l}$ appear as coefficients of the Taylor expansion [19]

$$
\hat{g}(q)=\sum_{l} \frac{(-i q)^{l}}{l !} M_{l} e^{-i q \mu} .
$$


By neglecting orders higher than the fourth and assuming negligible absorption (without loss of generality: $\left.M_{0} \approx 1\right), \hat{g}(q)$ reads

$$
\hat{g}(q) \approx \underbrace{\exp (-i q \mu)}_{\exp \left(i \phi_{s}\right)} \underbrace{\left(1-\frac{q^{2}}{2} M_{2}+i \frac{q^{3}}{6} M_{3}+\mathscr{O}\left(q^{4}\right)\right)}_{\exp \left(i \phi_{a}\right)} .
$$

According to eq. (4) there are two contributions to the value $P$ (i.e, $P=\phi_{s}+\phi_{a}$ ), which makes it clear that $P=\mu$ (with $q_{1}=1$ ) is only correct if in the absence of moments of higher order than two. The asymmetry of the scattering distribution is quantified by $M_{3}$ and its influence on $P$ can be seen at the definition of $\phi_{a}$

$$
\tan \phi_{a}=\frac{\frac{1}{6} q_{1}^{3} M_{3}}{1-\frac{1}{2} q_{1}^{2} M_{2}}
$$

Thus, an asymmetric scattering distribution leads to an additional systematic error in fringe offset value $P$, which is retrieved by eq. (2). Experiments showed that realistic values for $M_{3}$ are in the order of $10^{-2}$. Comparing the resulting $\phi_{a}\left(10^{-3}\right)$ to a detectable $\phi_{s}\left(10^{-2}\right)$ shows that $\phi_{a}$ constitutes a significant contribution to $P$.

In [18] we proposed that a deconvolution procedure in order to directly retrieve the scattering distribution $g(\phi)$. In principle, this approach avoids the occurrence of the presented systematic error. However, in the realistic case of present noise the accuracy of a deconvolution is affected, which constitutes a different contribution to the uncertainty of the phase signal. A comparison of the performance of both approaches will be an issue for future investigations.

\section{REFERENCES}

1. C. David, B. Nöhammer, H.H. Solak, and E. Ziegler, Appl. Phys. Lett. 81, 3287-3289 (2002).

2. A. Momose, S. Kawamoto, I. Koyama, Y. Hamaishi, K. Takai, and Y. Suzuki, Jpn. J. Appl. Phys. 42, L866-L868 (2003).

3. G. Schulz, T. Weitkamp, I. Zanette, F. Pfeiffer, F. Beckmann, C. David, S. Rutishauser, E. Reznikova, and B. Müller, J. Roy. Soc. Interface 7, 1665-76 (2010).

4. D. Stutman, T.J. Beck, J. Carrino, and C.O. Bingham, Phys. Med. Biol. 56, 5697-720 (2011).

5. M. Stampanoni, Z. Wang, T. Türing, C. David, E. Rössl, M. Trippel, R.A. Kubik-Huch, A. Rahel, G. Singer, M.K. Hohl, and N. Hauser, Inv. Rad. 46, 801-806 (2011).

6. F. Pfeiffer, O. Bunk, C. David, M. Bech, G. Le Duc, A. Bravin, and P. Cloetens, Phys. Med. Biol. 52, 6923-6930 (2007).

7. P. Cloetens, Contribution to Phase Contrast Imaging, Reconstruction and Tomography with Hard Synchrotron Radiation: Principles, Implementation and Applications, $\mathrm{PhD}$ thesis, Vrije Univeriteit Brussel, 1999.

8. T.J. Suleski, Appl. Optics 36, 4686-91 (1997).

9. T. Weitkamp, A. Diaz, C. David, F. Pfeiffer, M. Stampanoni, P. Cloetens, and E. Ziegler, Opt. Express 13, 6296-6304 (2005).

10. F. Pfeiffer, M. Bech, O. Bunk, P. Kraft, E.F. Eikenberry, Ch. Brönnimann, C. Grünzweig, and C. David, Nat. Mater. 7, 134-7 (2008).

11. Z. Wang, K.-J. Kang, Z.-F. Huang, and Z.-Q. Chen, Appl. Phys. Lett. 95, 094105 (2009).

12. A. Momose, W. Yashiro, Y. Takeda, Y. Suzuki, and T. Hattori, Jpn. J. Appl. Phys. 45, 5254-5262 (2006). 
13. S.A. McDonald, F. Marone, C. Hintermüller, G. Mikuljan, C. David, F. Pfeiffer, and M. Stampanoni, J. Synchrotron Radiat. 16, 562-72 (2009).

14. V. Revol, C. Kottler, R. Kaufmann, U. Straumann, and C. Urban, Rev. Sci. Instrum. 81, 073709 (2010).

15. P. Modregger, B.R. Pinzer, T. Thüring, S. Rutishauser, C. David, and M. Stampanoni, Opt. Express 19, 18324-18338 (2011).

16. K.J. Engel, D. Geller, T. Köhler, G. Martens, S. Schusser, G. Vogtmeier, and E. Rössl, Nucl. Instrum. and Meth. A 648, 202-207 (2010).

17. R. Raupach and T.G. Flohr, Phys. Med. Biol. 56, 2219-2244 (2011).

18. P. Modregger, F. Scattarella, B.R. Pinzer, C. David, R. Bellotti, and M. Stampanoni, Phys. Rev. Lett. 108, 048101 (2012).

19. F. James, Statistical Methods in Experimental Physics, 2nd ed. (World Scientific Publishing Co. Pte. Ltd., Singapore, 2006). 